\title{
Clinical ineffectiveness of latamoxef for Stenotrophomonas maltophilia infection
}

\author{
Hideharu Hagiya' \\ Ken Tasaka ${ }^{2}$ \\ Toshiaki Sendo ${ }^{2}$ \\ Fumio Otsuka' \\ 'Department of General Medicine, \\ Okayama University Graduate \\ School of Medicine, Dentistry and \\ Pharmaceutical Sciences, ${ }^{2}$ Department \\ of Pharmacy, Okayama University \\ Hospital, Okayama, Japan
}

Correspondence: Hideharu Hagiya Department of General Medicine, Okayama University Graduate School of Medicine, Dentistry and Pharmaceutical Sciences, 2-5-I Shikata-cho, Kitaku, Okayama 700-8558, Japan

$\mathrm{Tel}+8 \mathrm{I} 862357342$

Fax +8 I 862357345

Email e_dai_for_all@hotmail.com
This article was published in the following Dove Press journal:

Infection and Drug Resistance

20 October 2015

Number of times this article has been viewed

\begin{abstract}
Objectives: Stenotrophomonas maltophilia shows wide-spectrum resistance to antimicrobials and causes various infections in immunocompromised or critically ill patients with high mortality. In this era of antibiotics resistance, a revival of old antibiotics is now featured. We examined the clinical usefulness of latamoxef (LMOX) for the treatment of S. maltophilia infection.
\end{abstract}

Patients and methods: The observational study was retrospectively performed at Okayama University Hospital (Okayama, Japan) from January 2011 to December 2013. LMOX was administered to 12 patients with $S$. maltophilia infection, with eleven of those patients being admitted to the intensive care unit.

Results: Underlying conditions of the patients included postoperation, hematological transplantation, hepatic transplantation, and burn. Major infectious foci were surgical site infection ( ix cases), respiratory infection (four cases), blood stream infection (three cases), and burn site infection (one case). The doses of LMOX administered ranged from $1 \mathrm{~g} / \mathrm{d}$ to $3 \mathrm{~g} / \mathrm{d}$ for ten adult patients and from $40 \mathrm{mg} / \mathrm{kg} / \mathrm{d}$ to $80 \mathrm{mg} / \mathrm{kg} / \mathrm{d}$ for two pediatric patients. Microbiologic failure was seen in five $(41.7 \%)$ of 12 cases, and 30 -day and hospital mortality rates were $25 \%$ and $50 \%$, respectively. Minimum inhibitory concentrations of LMOX were higher in the deceased group $(4-64 \mu \mathrm{g} / \mathrm{mL})$ than in the surviving group $(1-4 \mu \mathrm{g} / \mathrm{mL})$.

Conclusion: LMOX treatment is not recommended for the treatment of S. maltophilia infection. Further investigation would be needed before its clinical use.

Keywords: latamoxef, Stenotrophomonas maltophilia, intensive care unit, revival

\section{Introduction}

Stenotrophomonas maltophilia is an emerging pathogen that characteristically causes severe nosocomial infections, particularly in immunocompromised or critically ill patients. ${ }^{1}$ The organism exhibits resistance to a broad array of antibiotics including carbapenems and yields high mortality rate. ${ }^{2-4}$ Although trimethoprim/sulfamethoxazole $(\mathrm{T} / \mathrm{S})$, tetracyclines, or fluoroquinolones (FQs) have been used for the treatment of S. maltophilia infection, the clinical effectiveness of these agents is limited and an emergence of new drugs potentially active against $S$. maltophilia has been coveted.

In recent years, the development of new antibiotics mainly depends on the modification of preexisting drugs, ${ }^{5}$ and the number of newly manufactured drugs is decreasing. ${ }^{6}$ In this antibiotics resistance era, thus, a revival of old drugs can be a solution. Actually, fosfomycin and colistin are considered to be representatives of such agents. ${ }^{7,8}$

Since latamoxef (LMOX) had first appeared on the market in 1981, the drug has been administered in various situations. ${ }^{9}$ However, the frequency of its clinical use has 
been declining with the development of other antibiotics. In our medical facility, LMOX has been administered to patients suffering from $S$. maltophilia infection under a recommendation by the Department of Infectious Disease. LMOX could be a candidate for such a revival use; however, the evidence of clinical effectiveness of LMOX for S. maltophilia infection is scarce. Only in vitro study has been reported, historically. ${ }^{10,11}$ The authors consider that the effectiveness of LMOX for S. maltophilia infection should be carefully examined before its clinical use. To evaluate and discuss its clinical utility, we summarized previous cases in our facility.

\section{Patients and methods}

This is a retrospective study performed at Okayama University Hospital to investigate the potential usefulness of LMOX for the treatment of $S$. maltophilia infection. The present study was approved by the Institutional Review Board of the facility (No 762). Study period was set between 2011 and 2013. Data were extracted from electronic medical records obtained from the database at the Department of Pharmacy. The patients were those who had received LMOX for $S$. maltophilia infection. In our medical facility, the administration of LMOX was strictly controlled by the Department of Infectious Diseases, and the drug cannot be ordered without permission. Any of the authors were not in charge for clinical decision in choosing antimicrobials. Data for clinical background (age, sex, admission place, and underlying diseases), laboratory and microbiological data (specimens and primary infectious focus), antimicrobial treatment (dose of LMOX and duration of administration), and prognosis of the patients were collected and analyzed. Sequential organ failure assessment (SOFA) score was evaluated on the day when LMOX treatment was initiated. Microbiologic failure was defined as a positive result of bacterial culture for S. maltophilia while administering LMOX to patients. Primary outcome was set as the prognosis of patients. Statistical analysis was performed by Mann-Whitney's $U$-test to analyze numerical data by using SPSS software (version 17.0 for Windows; SPSS Inc., Chicago, IL, USA). A $P$-value $<0.05$ was considered to be significant.

\section{Results}

Characteristics of the patients and clinical outcomes are shown in Table 1. Patients who died during the clinical course were classified as the dead group (Cases 1-6), and those who survived were classified as the surviving group (Cases 7-12). The two groups consisted of 12 patients (eleven men and one woman) with a mean age of $52.8 \pm 27.1$ years (ranging from 1 year to 81 years). All but one patient (11/12 cases) were admitted to the intensive care unit (ICU). Major underlying conditions were hematological transplantation (three cases), cardiac surgery (three cases), abdominal surgery (three cases), hepatic transplantation (one case), and burn (one case). Bone marrow transplantation (BMT) was performed in three cases (Cases 2, 4, and 5). Four (33.3\%) of the 12 patients $(33.3 \%)$ were in a state of neutropenia. The average SOFA score for the eleven ICU patients was $10.7 \pm 2.6$ (ranging from 8 to 15). The patient in a general ward was in a postoperative state following orthopedic surgery.

S. maltophilia was detected from sputum (eight cases), blood (five cases), ascites (four cases), surgical site (three cases), and pleural effusion (one case). The primary infectious foci were surgical site infection (six cases), respiratory infection (four cases), blood stream infection (three cases), and burn site infection (one case). The doses of LMOX administered ranged from $1 \mathrm{~g} / \mathrm{d}$ to $3 \mathrm{~g} / \mathrm{d}$ for adult patients and from $40 \mathrm{mg} / \mathrm{kg} / \mathrm{d}$ to $80 \mathrm{mg} / \mathrm{kg} / \mathrm{d}$ for pediatric patients. Eleven patients (91.7\%) were administered LMOX combined with other antimicrobials. Significant adverse effects of LMOX were unapparent. The 30-day mortality rate was $25 \%$ (3/12 cases), and hospital mortality rate was $50 \%$ (6/12 cases).

Comparison of the dead and surviving groups was as follows. Mean age was not significantly different (50.7 years in the dead group vs 55 years in the surviving group; $P=0.937$ ). The patients in both groups had various underlying conditions. All the patients who had undergone BMT were in the dead group. SOFA score tended to be higher in the dead group, but there was no significant difference (11.3 vs 10 ; $P=0.931$ ). There were various primary infectious foci in both groups. However, bacteremia and microbiologic failure were more common in the dead group than in the surviving group (four cases vs one case).

The results of antibiotics susceptibility testing are summarized in Table 2. Susceptibility testing for minocycline (MINO), levofloxacin, and ceftazidime (CAZ) was performed in all cases but that for $\mathrm{T} / \mathrm{S}$ was performed in only eight cases (66.7\%). Susceptible testing of ticarcillin-clavulanate was not performed since the antibiotic is unavailable in Japan. Based on breakpoints defined by the Clinical and Laboratory Standards Institute (CLSI, M100-S23), susceptibility ratios of the antibiotics were $100 \%$ for T/S (8/8), 100\% for MINO $(12 / 12), 58.3 \%$ for levofloxacin (7/12), and $25 \%$ for CAZ (3/12). Minimum inhibitory concentrations of LMOX were found to be higher in the dead group (4-64 $\mu \mathrm{g} / \mathrm{mL}$ ) than in the surviving group $(1-4 \mu \mathrm{g} / \mathrm{mL})$. 


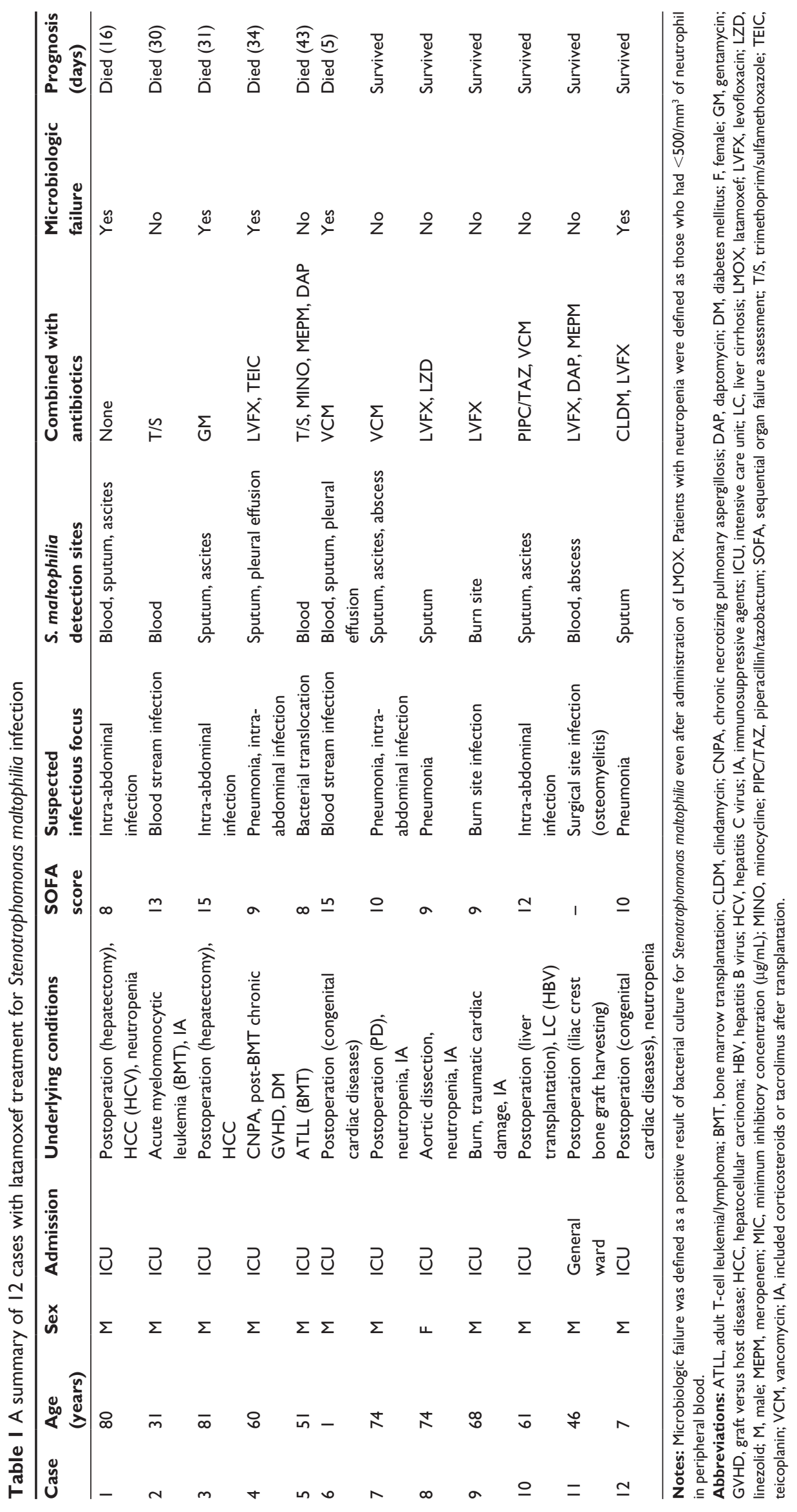


Table 2 Results of antibiotics susceptibility testing $(\mu \mathrm{g} / \mathrm{mL})$

\begin{tabular}{|c|c|c|c|c|c|}
\hline Case & $\mathbf{T} / \mathbf{S}$ & MINO & LVFX & CAZ & LMOX \\
\hline $\mathrm{I}$ & $\mathrm{np}$ & 2 & 8 & 4 & $\mathrm{np}$ \\
\hline 2 & $0.125 / 2.375$ & I & 8 & $>16$ & $8-16$ \\
\hline 3 & $1 / 19$ & 4 & $>8$ & $>16$ & $8-64$ \\
\hline 4 & $0.125 / 2.375$ & $\leq 0.5$ & 0.5 & $>16$ & 4 \\
\hline 5 & $1 / 19$ & $\leq 0.5$ & 2 & $>16$ & $4-8$ \\
\hline 6 & $1 / 19$ & $\leq 0.5$ & 0.5 & $>16$ & 32 \\
\hline 7 & $\mathrm{np}$ & I & 8 & 16 & $\mathrm{np}$ \\
\hline 8 & np & $\leq 0.5$ & I & 8 & $2-4$ \\
\hline 9 & $\mathrm{np}$ & $\leq 0.5$ & 2 & $>16$ & $\mathrm{np}$ \\
\hline 10 & $0.25 / 4.75$ & $\leq 0.5$ & 8 & I & $\mathrm{I}-4$ \\
\hline 11 & $2 / 38$ & $\leq 0.5$ & 2 & I & $\mathrm{I}-4$ \\
\hline 12 & $2 / 38$ & $\leq 0.5$ & I & 16 & 4 \\
\hline
\end{tabular}

Notes: Minimum inhibitory concentrations $(\mu \mathrm{g} / \mathrm{mL})$ are shown. Antibiotic susceptibility testing was performed according to Clinical and Laboratory Standards Institute (MI00-S23). Antimicrobials that were sensitive to pathogenic organisms in each case are displayed in bold. Breakpoint of LMOX to Stenotrophomonas maltophilia is not defined, but those for patients who survived (Cases 7-I2) were $<4 \mu \mathrm{g} / \mathrm{mL}$.

Abbreviations: CAZ, ceftazidime; LMOX, latamoxef; LVFX, levofloxacin; MINO, minocycline; $n p$, not performed; $T / S$, trimethoprim/sulfamethoxazole.

\section{Discussion}

With respect to the clinical effectiveness of LMOX for the treatment of $S$. maltophilia infection, the present study did not show a satisfactory outcome. Since the clinical conditions of the patients were comparatively complicated, confounding factors for prognosis must have existed. Due to the small number of cases, such factors have not been adjusted in this study. However, in this era of antibiotic resistance, consideration for revivals of "forgotten antibiotics" as this study would be meaningful. In this respect, though small scaled and retrospective, the present study is valuable.

Eleven $(91.7 \%)$ out of the 12 patients were ICU patients, and most of them had various predisposing conditions. Notably, four of the 12 patients had received solid organ transplantation or BMT, and the other four were in a state of neutropenia. Four patients were administered immunosuppressive agents. It has been reported that $>90 \%$ of patients with $S$. maltophilia infection have some risk factors, ${ }^{12}$ such as malignancy, immunocompromised host, long-term hospitalization, presence of indwelling devices, use of carbapenems, ICU admission, and breakdown of mucocutaneous defense barriers. ${ }^{13}$ Thus, in terms of clinical backgrounds, our cases were compatible with those in the previous reports. On the other hand, the major primary infectious focus in the present study was surgical site infection, while respiratory infection and bacteremia were common in the previous studies. ${ }^{2}$

In general, we chose antibiotics based on the infectious foci and results of antimicrobial susceptibility testing. CLSI or European Committee on Antimicrobial Susceptibility
Testing (EUCAST) sets breakpoints of each antimicrobial against various pathogens. Without these criteria, it would be difficult for clinicians to determine the appropriate antimicrobials. T/S, MINO, FQs, CAZ, and ticarcillinclavulanate are currently considered the choice for the treatment of S. maltophilia infection., ${ }^{2,12}$ Among them, although resistant strains have been reported to be increasing, ${ }^{15} \mathrm{~T} / \mathrm{S}$ is considered as a first-choice drug. ${ }^{2}$ However, the antibiotic susceptibility testing for $\mathrm{T} / \mathrm{S}$ was not performed in four of the 12 cases in the present study. The authors consider that antimicrobial susceptible testing of this clinically important antibiotic agent should have been performed in all cases.

As for LMOX, on the other hand, neither CLSI nor EUCAST has set a breakpoint against any pathogens including $S$. maltophilia. In addition, usual and renal dose of LMOX remains undetermined. It has been reported that LMOX shows a low minimum inhibitory concentration against $S$. maltophilia in vitro, ${ }^{10,11}$ but there is little clinical experience for the treatment in $S$. maltophilia infection. Insufficient data in its clinical use would be the main reason for this drug not being recommended by those authoritative organizations. Rather, other beta-lactams such as CAZ or ticarcillin-clavulanate are often of choice. An effectiveness of FQs has been increasingly reported. ${ }^{16,17}$

In our study, beneficial efficacy of LMOX for the treatment of $S$. maltophilia infection was not shown. Microbiologic failure was seen in five (41.7\%) of 12 cases, and the hospital mortality rate was 50\%. Limitations include being not a comparative study and small patient population with heterogeneous backgrounds and therapeutic schemes in terms of LMOX dosing and antimicrobial combination. Following basic study, a cohort study with comparison to other antibiotics is necessary to make a conclusion regarding the clinical effectiveness of LMOX. At present, LMOX is not allowed for the treatment of $S$. maltophilia infection.

\section{Disclosure}

The authors report no conflicts of interest in this work.

\section{References}

1. Friedman ND, Korman TM, Fairley CK, Franklin JC, Spelman DW. Bacteraemia due to Stenotrophomonas maltophilia: an analysis of 45 episodes. J Infect. 2002;45(1):47-53.

2. Brooke JS. Stenotrophomonas maltophilia: an emerging global opportunistic pathogen. Clin Microbiol Rev. 2012;25(1):2-41.

3. Jang TN, Wang FD, Wang LS, Liu CY, Liu IM. Xanthomonas maltophilia bacteremia: an analysis of 32 cases. Journal of the Formosan Medical Association. 1992;91(12):1170-1176.

4. Victor MA, Arpi M, Bruun B, Jonsson V, Hansen MM. Xanthomonas maltophilia bacteremia in immunocompromised hematological patients. Scand J Infect Dis. 1994;26(2):163-170. 
5. Bush K. Improving known classes of antibiotics: an optimistic approach for the future. Curr Opin Pharmacol. 2012;12(5):527-534.

6. Boucher HW, Talbot GH, Benjamin DK Jr, et al; Infectious Diseases Society of America. $10 \times$ ' 20 Progress - development of new drugs active against gram-negative bacilli: an update from the Infectious Diseases Society of America. Clin Infect Dis. 2013;56(12):1685-1694.

7. Michalopoulos AS, Livaditis IG, Gougoutas V. The revival of fosfomycin. Int J Infect Dis. 2011;15(11):e732-e739.

8. Dhariwal AK, Tullu MS. Colistin: re-emergence of the 'forgotten' antimicrobial agent. J Postgrad Med. 2013;59(3):208-215.

9. Carmine AA, Brogden RN, Heel RC, Romankiewicz JA, Speight TM, Avery GS. Moxalactam (latamoxef). A review of its antibacterial activity, pharmacokinetic properties and therapeutic use. Drugs. 1983;26(4):279-333.

10. Akova M, Bonfiglio G, Livermore DM. Susceptibility to beta-lactam antibiotics of mutant strains of Xanthomonas maltophilia with high- and low-level constitutive expression of L1 and L2 beta-lactamases. $J$ Med Microbiol. 1991;35(4):208-213.

11. Lecso-Bornet M, Pierre J, Sarkis-Karam D, Lubera S, Bergogne-Berezin E. Susceptibility of Xanthomonas maltophilia to six quinolones and study of outer membrane proteins in resistant mutants selected in vitro. Antimicrob Agents Chemother. 1992;36(3):669-671.
12. Gulmez D, Hascelik G. Stenotrophomonas maltophilia: antimicrobial resistance and molecular typing of an emerging pathogen in a Turkish university hospital. Clin Microbiol Infect. 2005;11(11):880-886.

13. Lai $\mathrm{CH}$, Chi CY, Chen HP, et al. Clinical characteristics and prognostic factors of patients with Stenotrophomonas maltophilia bacteremia. J Microbiol Immunol Infect. 2004;37(6):350-358.

14. Jones RN, Sader HS, Beach ML. Contemporary in vitro spectrum of activity summary for antimicrobial agents tested against 18569 strains non-fermentative Gram-negative bacilli isolated in the SENTRY Antimicrobial Surveillance Program (1997-2001). Int J Antimicrob Agents. 2003;22(6):551-556.

15. Muder RR. Optimizing therapy for Stenotrophomonas maltophilia. Semin Respir Crit Care Med. 2007;28(6):672-677.

16. Cho SY, Kang CI, Kim J, set al. Can levofloxacin be a useful alternative to trimethoprim-sulfamethoxazole for treating Stenotrophomonas maltophilia bacteremia? Antimicrob Agents Chemother. 2014;58(1): 581-583.

17. Wang YL, Scipione MR, Dubrovskaya Y, Papadopoulos J. Monotherapy with fluoroquinolone or trimethoprim-sulfamethoxazole for treatment of Stenotrophomonas maltophilia infections. Antimicrob Agents Chemother. 2014;58(1):176-182.
Infection and Drug Resistance

\section{Publish your work in this journal}

Infection and Drug Resistance is an international, peer-reviewed openaccess journal that focuses on the optimal treatment of infection (bacterial, fungal and viral) and the development and institution of preventive strategies to minimize the development and spread of resistance. The journal is specifically concerned with the epidemiology of antibiotic

\section{Dovepress}

resistance and the mechanisms of resistance development and diffusion in both hospitals and the community. The manuscript management system is completely online and includes a very quick and fair peerreview system, which is all easy to use. Visit http://www.dovepress.com/ testimonials.php to read real quotes from published authors. 